\title{
Current sheets from Ulysses observation
}

\author{
B. Miao ${ }^{1,3}$, B. Peng ${ }^{2}$, and G. $\mathrm{Li}^{1,2}$ \\ ${ }^{1}$ CSPAR, University of Alabama in Huntsville, Huntsville, AL 35805, USA \\ ${ }^{2}$ Department of Physics, University of Alabama in Huntsville, Huntsville, AL 35805, USA \\ ${ }^{3}$ CAS Key Laboratory of Basic Plasma Physics, School of Earth and Space Sciences, University of Science and Technology \\ of China, Hefei, Anhui 230026, China
}

Received: 1 October 2010 - Revised: 5 January 2011 - Accepted: 25 January 2011 - Published: 2 February 2011

\begin{abstract}
Current sheet is a significant source of solar wind MHD turbulence intermittency. It has long been recognized that these structures can arise from non-linear interactions of MHD turbulence. Alternatively, they may also be relic structures in the solar wind that have a solar origin, e.g., magnetic walls of flux tubes that separate solar wind plasma into distinct parcels. Identifying these structures in the solar wind is crucial to understanding the properties of the solar wind MHD turbulence. Using Ulysses observations we examine 3-year worth of solar wind magnetic field data when the Ulysses is at low latitude during solar minimum. Extending the previous work of $\operatorname{Li}(2007,2008)$, we develop an automatic data analysis method of current sheet identification. Using this method, we identify more than 28000 current sheets. Various properties of the current sheet are obtained. These include the distributions of the deflection angle across the current sheet, the thickness of the current sheet and the waiting time statistics between current sheets.
\end{abstract}

Keywords. Solar physics, astrophysics, and astronomy (Magnetic fields) - Space plasma physics (Discontinuities; Turbulence)

\section{Introduction}

Magnetohydrodynamics (MHD) turbulence has been a central topic of space plasma physics (see Tu and Marsch, 1995; Goldstein et al., 1995; Bruno and Carbone, 2005, for extensive reviews). Because of the presence of a strong magnetic field, MHD turbulence differs in many aspects from the hydrodynamic turbulence (Iroshnikov, 1964; Kraichnan, 1965; Biskamp, 1993). Various technical difficulties have made studying of MHD turbulence in terrestrial laboratories

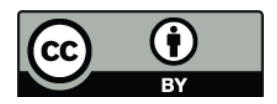

Correspondence to: $\mathrm{G} . \mathrm{Li}$ (gang.li@uah.edu) very difficult. In particular, studying the dynamical evolution of MHD turbulence in terrestrial laboratory experiments is difficult because these experiments are short lived. In comparison, solar wind provides a natural laboratory to study MHD turbulence in a collisionless plasma. In particular, the launches of multiple spacecraft in the past several decades, noticeably Voyager, Helios, WIND, Ulysses and Cluster, have accumulated a significant amount of plasma and magnetic field data These data have revealed valuable information about MHD turbulence and its dynamical evolution.

A central topic of the solar wind MHD turbulence is intermittency. In a collisionless plasma such as the solar wind, intermittency arises because the fluctuations of magnetic field or fluid velocity are not scale invariant as conjectured in the first hydrodynamic turbulence theory Kolmogorov (1941) (hereafter K41 theory). Roughly speaking, intermittency reflects how turbulence is unevenly distributed in space. Mathematically, intermittency describes how a structure function $S_{q}^{p}(l)$ varies with the order $p$. Here $S_{q}^{p}(l)$ is the $p$-th order structure function defined for a physical quantity $q$ ( $q$ can be e.g. $v_{\|}$or $B$ of solar wind measurement) through,

$S_{q}^{p}(l)=<|q(x)-q(x+l)|^{p}>\approx l^{\zeta p}$.

In the above, the quantity $\zeta_{p}$ is the scaling exponent of $S_{q}^{p}(l)$ and is in general a function of $p$. In the absence of intermittency, $\zeta_{p}=p / m$ where $m=3$ for normal fluid and 4 for magnetofluid. Any deviation from this linear dependence indicates the presence of intermittency.

In the context of solar wind MHD turbulence, systematic study of intermittency was first done by Burlaga. In a series of papers (Burlaga, 1991a,b,c), using Voyager data at various heliocentric distances, Burlaga showed that the $\zeta_{p}$ associated with fluctuating solar wind speed is not linear with p. Marsch and Liu (1993) analyzed Helios data in the inner heliosphere and showed that not only intermittency exists in the solar wind, but its strength can also differ much

Published by Copernicus Publications on behalf of the European Geosciences Union. 
depending on plasma properties: small scales are more intermittent than large scales and slow wind is more intermittent than fast wind. Since the work of Marsch and Liu (1993), studies on the intermittent character of solar wind have been widely reported (e.g. Marsch and Tu, 1994, 1997; Carbone et al., 1995a,b; Ruzmaikin et al., 1995; Tu et al., 1996; Horbury et al., 1996, 1997; Bruno et al., 1999, 2001, 2003, 2004; Veltri et al., 2005; Salem et al., 2007, 2009). Relating intermittency with specific solar wind turbulence models has been reported by Ruzmaikin et al. (1995). In this paper, the authors showed that if one reduces the measured spectral index of magnetic field fluctuations by an amount governed by the intermittency scaling exponent, then the reduced power spectral index will yield a scaling agreeing with the randomphase Alfvénic turbulence model of Kraichnan (1965). Later, Tu et al. (1996) tried to integrate the $p$-model of Meneveau and Sreenivasan (1987) to the Tu (1988) model of a developing solar wind. Clearly, these works suggested that to understand the solar wind turbulence a good understanding of the solar wind MHD intermittency is necessary.

Observationally, a very important intermittent structure in the solar wind is current sheet. A current sheet is a 2-D structure where the magnetic field direction changes significantly from one side to the other. Using a Haar wavelets technique and magnetic field and fluid velocity data from ISEE space experiment, Veltri and Mangeney (1999) calculated solar wind power spectra and structure functions for a time range between $1 \mathrm{~min}$ to about 1 day. They found that in solar wind (a magneto-fluid) the most intermittent structures are shocks and current sheets where magnetic field rotates by an angle of about 120-130 degrees. This differs from ordinary fluids where the most intermittent structures are two-dimensional vortices. Veltri and Mangeney (1999) also pointed out that by using a conditional sampling scheme, one can eliminate the intermittency effects in the power spectra of the turbulence. Such studies therefore can provide a possible distinguishment between Kolmogorov type cascading and Kraichnan type cascading in the solar wind.

Later Bruno et al. (2001) studied current sheets using Helios 2 data at $0.9 \mathrm{AU}$. They performed a minimum variance analysis to study how the solar wind magnetic field vector evolves for several selected time periods. By plotting the trajectory of the tip of the magnetic field vector in the minimum variance reference system, Bruno et al. (2001) showed that the magnetic field direction at times undergo abrupt changes, implying the presence of current sheet. Furthermore, Bruno et al. (2001) have also proposed the possibility that most of these flux tubes might be of solar origin and, as such, advected by the wind. The work by Bruno et al. (2001) was the first to suggest that current sheets in the solar wind could be the borders between adjacent flux tubes. By examining the waiting time statistics, these same authors go even one more step and estimated that the size of those possible tubes observed at $1 \mathrm{AU}$ when projected back onto the Sun would have cross sections not far from the average size of photo- spheric structures, making a remarkable connection between structures in the solar wind and those on the surface of the Sun.

While the analysis of Bruno et al. (2001) showed that current sheets are common in the solar wind and they may be the boundaries of flux tubes, there are alternative views about the origin of current sheets. For example, numerical MHD simulations by Zhou et al. (2004) showed that current sheets emerge as the dynamical evolution of the nonlinear interactions of the solar wind MHD turbulence, i.e. the generation of current sheet can be spontaneous. Similarly study by Chang et al. (2004) also showed that starting from an isotropic initial MHD turbulence state, non-linear interactions in the solar wind can lead to the emergence of various coherent structures, including current sheet. These studies (Zhou et al., 2004; Chang et al., 2004) suggested that current sheet is an intrinsic property of the solar wind MHD turbulence. In contrast, advocating Bruno et al. (2001)'s idea, Borovsky (2008), on examining one-year worth magnetic field data from the ACE spacecraft, has found a clear signature of two population of current sheets with one extending to large angle separations. Borovsky (2008) suggested that these current sheets are the "magnetic walls" of flux tubes in the solar wind and they are relic structures which can be traced back to the surface of the Sun. In this picture, current sheets are carried out by the solar wind as passive structures. The plasma in the solar wind are bundled in "spaghetti-like" flux tubes. Such a picture is consistent with some old ideas proposed over 40 years ago. Indeed, solar wind being consist of "spaghettilike" flux tubes has been suggested by Bartley et al. (1966) and McCracken and Ness (1966) as an attempt to explain the modulation of cosmic rays and later adopted by Mariani et al. (1973) to explain the observed variations in the occurrence rate of discontinuities in interplanetary magnetic field. The suggestion of Bruno et al. (2001) and later Borovsky (2008) is interesting because in this picture, flux tubes will introduce an extra source of intermittency besides that generated by non-linear interactions such as shown by Zhou et al. (2004). Since this intermittency is not intrinsic to the solar wind MHD turbulence, one has to pay extra attention to these structures in understanding the properties of the solar wind MHD turbulence.

Extending the work of Borovsky (2008), Li (2008) developed a systematic method to identify current sheets in the solar wind. The essence of the method is to study the $\zeta$ scaling properties of the angle $\theta=\cos ^{-1}(\hat{B}(t) \cdot \hat{B}(t+\zeta))$. This method allows one to show statistically the existence of current sheets. Li (2008) further presented a method to obtain the exact location of individual current sheets. Applying this method to magnetic field data from Cluster spacecraft, Li et al. (2008) attempted to answer the question of "are there current sheets like those in the solar wind in the Earth's magnetotail?" Obviously, there is no structures similar to supergranules on the solar surface in the Earth's magnetosphere. Therefore if there are similar current sheets exist 
in the Earth's magnetotail as in the solar wind, then these current sheets are generated by non-linear interactions of MHD turbulence. If however, no such current sheets are found in the Earth's magnetotail, then it suggests that current sheets in the solar wind may be the relic structures originated from the surface of the Sun. For two selected periods, using the same instrument on Cluster, $\mathrm{Li}$ et al. (2008) found that there is no clear signature of current sheets in the Earth's magnetosphere, but there are clear signatures of current sheets in the solar wind. Therefore the study of Li et al. (2008) is consistent with the proposal advocated by Bruno et al. and Borovsky.

We note here that the plasma environment of the Earth's magnetotail is different from the solar wind. The MHD turbulence of the magnetotail may not be as fully developed as that in the solar wind at $1 \mathrm{AU}$. Consequently, there may be fewer current sheets emerging in the Earth's magnetotail. However, it has been argued in (Chang, 1999) that coherent flux tubes do exist in the Earth's magnetotail and local reconnections among them are the origin of the observed "bursty bulk flows".

The above discussion illustrates the importance of developing an accurate data analysis method to identify individual current sheets in the solar wind. Only with such analysis methods available, could we understand the solar wind MHD turbulence intermittency. In this work, we extend the method proposed in $(\mathrm{Li}, 2007,2008)$ and develop an automatic current sheet identification procedure. We then apply this procedure to a 3-year worth Ulysses magnetic field data. More than 28000 current sheets are identified. The properties of these current sheets, including the distributions of current sheet width and deflection angle, and the waiting time analysis between current sheets are obtained.

The paper is organized as the following: we first briefly discuss the technique used in $(\mathrm{Li}, 2007,2008)$ in Sect. 2. We then present our period selection and the corresponding data analysis in Sect. 3. We conclude in Sect. 4.

\section{Observation of current sheets by the ULYSSES}

\subsection{Data selection}

It is ideal to select data in the solar minimum period for studying current sheet events because transient disturbance, e.g. CMEs are relatively inactive during the solar minimum. Therefore in this study we use magnetic field measurements from the Ulysses VHM/FGM (Balogh et al., 1992) instrument in the solar minimum years. In addition, we restrict our data selection to low latitude. One reason for doing so is that in this study we want to focus on slow solar wind and avoid fast solar wind at high latitude. Of course, fast wind streams are inevitable at low latitudes, and as will be seen from our results, the current sheet occurrence rate does show correlation with the solar wind speed.
Data is taken when the Ulysses spacecraft was within $30^{\circ}$ of the heliosphere ecliptic plane. With these criteria, two periods are identified. One from the day 300 in 1996 to the day 365 in 1997, and the other from the day 1 in 2004 to day 3 in 2006. The data selections are illustrated in Fig. 1, in which the regions $\mathrm{A}$ and $\mathrm{B}$ are marked. In Fig. 1, magnitude of the total magnetic field $B_{\mathrm{t}}$, solar wind speed $V_{\mathrm{sw}}$, heliocentric distance $R$, latitude Lat, and longitude Lon of the Ulysses spacecraft, and the number of the sunspots as functions of time are plotted from the top to the bottom, respectively. During the selected periods, the Ulysses VHM/FGM instrument returned most of the time 2-s resolution magnetic field data, and occasionally 1-s resolution data.

\subsection{Locating current sheet}

If the solar wind are structured and bundled as flux tubes, one would expect the change of the magnetic field direction between adjacent flux tubes be larger than that within the same flux tube due to the intrinsic turbulence. In this picture the current sheets are recognized as the boundaries of adjacent flux tubes. If the current sheets are generated in-situ as a signature of solar wind MHD turbulence intermittency, as suggested in Zhou et al. (2004) and Chang et al. (2004), the magnetic field direction will also change significantly across these structures. Therefore we expect, in both cases, current sheet crossing will correlate with an abrupt change of the magnetic field direction.

A statistical method to verify the existence of current sheets in the solar wind is to study the two-point correlation function $(\mathrm{Li}, 2008), R^{\mathcal{P}}(\zeta)=<\mathcal{P}(t) \mathcal{P}(t+\zeta)>$ and its $\zeta$ dependence. In the method of $(\mathrm{Li}, 2008)$, in order to show the existence of current sheet, a quantity called integrated distribution function $F(\theta, \zeta)$ is considered. For a time series of (unit) magnetic field data $\left\{\hat{b}\left(t_{1}\right), \hat{b}\left(t_{2}\right), \ldots, \hat{b}\left(t_{N}\right)\right\}, F(\theta, \zeta)$ is computed as the following,

$F(\theta, \zeta)=\frac{N^{\zeta}\left(\theta<\theta^{\prime}<\pi\right)}{N^{\zeta}\left(0<\theta^{\prime}<\pi\right)}$.

where $N^{\zeta}\left(\theta<\theta^{\prime}<\theta+\delta \theta\right)$ is the number of measurement pairs where the angle between $\hat{b}(t)$ and $\hat{b}(t+\zeta)$ is within the range of $(\theta, \theta+\delta \theta)$ and $N^{\zeta}\left(0<\theta^{\prime}<\pi\right)$ is the total number of measurements. Clearly $F(\theta, \zeta)$ represents the frequency of having the measured angle, between two unit magnetic field vector with a time separation of $\zeta$, larger than $\theta$.

Because we expect the angle between two magnetic field vectors that lie in different flux tubes is larger than the angle between two magnetic field vector within the same flux tube, one can define a critical angle $\theta_{0}$ that separates the angle between the magnetic field vectors within one flux tube and those between two adjacent flux tubes. It was shown in $\mathrm{Li}$ (2008) that the existence of the current sheets lead to the following scaling for the integrated distribution function $F(\theta, \zeta)$ :

$F(\theta, m \zeta) \sim m F(\theta, \zeta) \quad$ when $\quad \theta>\theta_{0}$. 


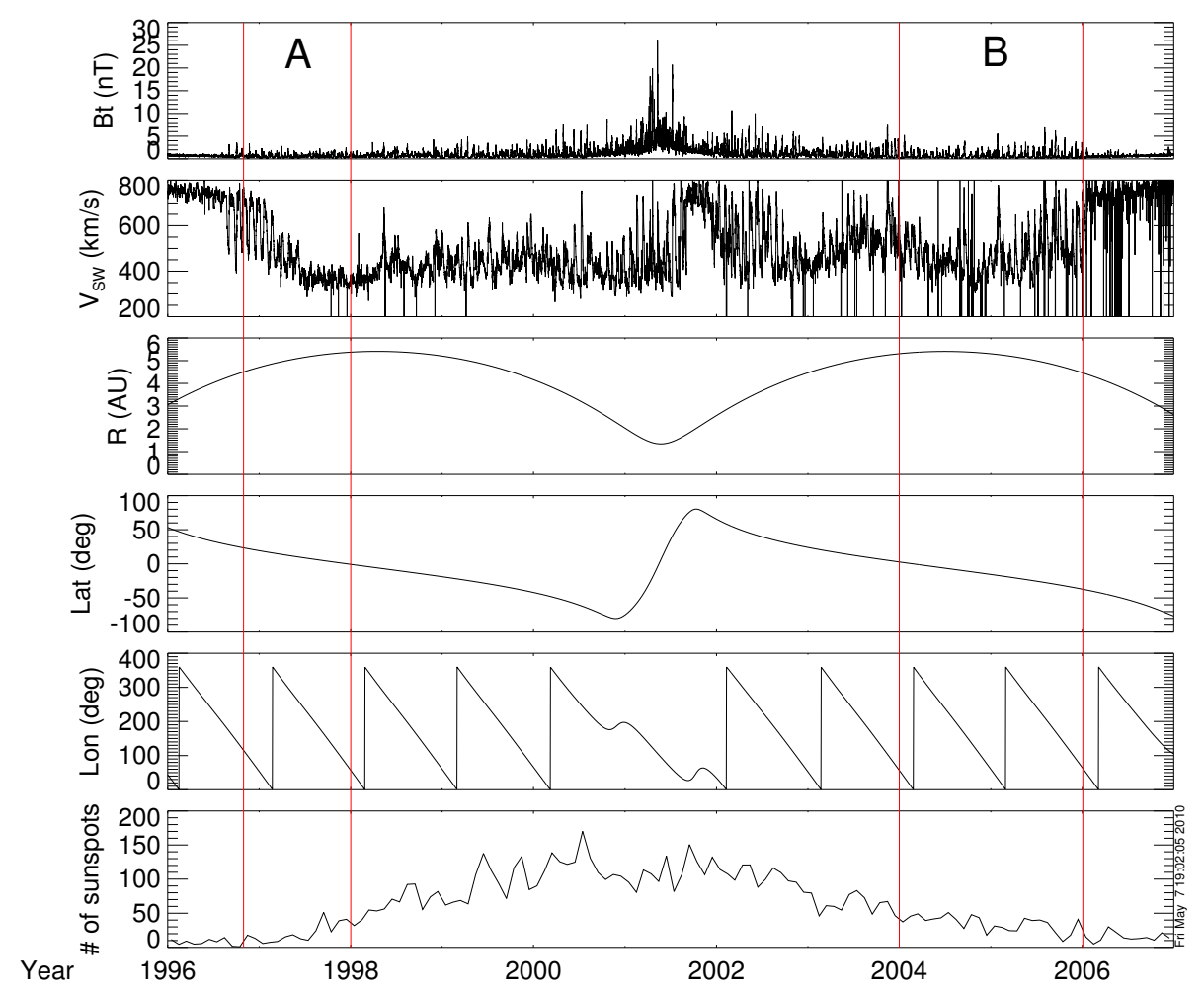

Fig. 1. Ulysses observations of the solar wind magnetic field $B_{\mathrm{t}}$, and the solar wind speed $V_{\mathrm{SW}}$ as well as the locations of the spacecraft, given by the heliocentric distance $R$, the latitude Lat and the longitude Lon. The number of sunspots is plotted in the bottom panel (from Royal Greenwich Observatory - USAF/NOAA Sunspot Data). Time period is from 1996-001 to 2006-365. The regions A and B used in this work are marked by the red lines.

This $\zeta$-scaling property of $F(\theta, \zeta)$ when $\theta>\theta_{0}$ provides an easy way to verify the existence of current sheet in the solar wind. It, however, requires to pre specify the quantity $\theta_{0}$ and the exact location of individual current sheets can not be obtained.

$\mathrm{Li}$ (2008) also provided a way to find the exact location of the current sheet. The essence is the following: when a current sheet crosses the spacecraft, the duration $\tau$ of having a continuous measurement of $\theta>\theta_{0}$ has approximately the same scaling law as $F(\theta, m \zeta)$, i.e.,

$\tau\left(m \zeta, \theta_{0}<\theta<\pi\right) \sim m \tau\left(\zeta, \theta_{0}<\theta<\pi\right)$.

Therefore, by examining the angle

$\theta(\zeta, t)=\cos ^{-1}(\hat{b}(t-\zeta / 2) \cdot \hat{b}(t+\zeta / 2))$

and search for a period of $\tau\left(\zeta, \theta_{0}<\theta<\pi\right)$ which linearly scale with $\zeta$, one can obtain the occurrence time of the current sheet $t$. It must be noted that due to the presence of intrinsic turbulence in the solar wind, within the duration $\tau$, it is possible that a few measurements of $\theta$ are smaller than $\theta_{0}$. Furthermore, a current sheet has a finite thickness, therefore the measured $\theta$ may increase gradually, instead of abruptly, from below $\theta_{0}$ to above $\theta_{0}$. Because of these, the scaling property for $\tau\left(\zeta, \theta_{0}<\theta<\pi\right)$ is less accurate than that for
$F(\theta, \zeta)$ and in this sense we term it as approximately satisfied. Finally, we note that the deflection angle, $\Delta \theta$, i.e., the angle between the background magnetic field directions of two adjacent flux tubes can vary from one current sheet to another, so to pre-specify a value for $\theta_{0}$, as done in $\mathrm{Li}$ (2008), can lead to missing those current sheets with a magnetic field deflection angle $\Delta \theta$ smaller than $\theta_{0}$. In this work, we address these issues and develop an automatic pattern search routine to identify individual current sheets which yield both the thickness $d$ and the deflection angle $\Delta \theta$ of the current sheet at the same time.

In Fig. 2, based on Eq. (5), the measurements of the angle $\theta(\zeta, t)$ as a function of time $t$ are shown for four different cases. In all four cases, three selected $\zeta$ 's, $24 \mathrm{~s}, 48 \mathrm{~s}$, and $96 \mathrm{~s}$ are used to identify the current sheet. All cases in the figure show significant increases of $\theta$ above the background value, and are seen to last a certain duration $\tau$. In all cases, $\tau$ scales with $\zeta$ as indicated by Eq. (4). As expected, different duration of $\tau \mathrm{s}$ also show approximately the same center positions. Note, the angle $\theta$ s increase "gradually" instead of "abruptly" to a maximum value. This rising period reflects the time period for the current sheet to pass the spacecraft, i.e. the thickness of the current sheet; the maximum value of $\theta$ is approximately the deflection angle $\Delta \theta$ between the 

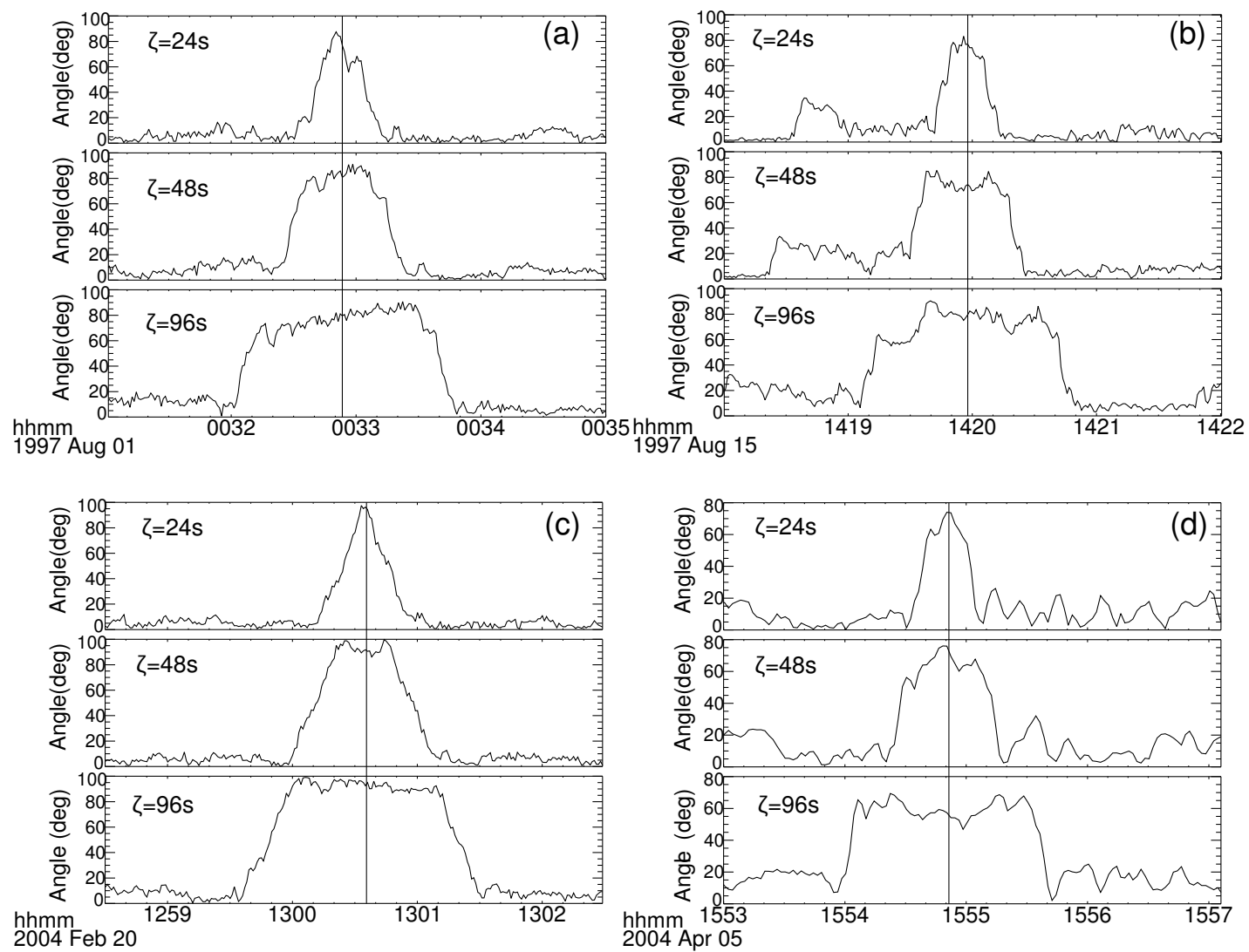

Fig. 2. Four cases to show the current sheet searching procedure. In each case, $\Delta \theta$ as a function of $\zeta$ and $t$ are shown for three different $\zeta$ 's: $\zeta=24,48$ and $96 \mathrm{~s}$. The $\mathrm{x}$-axis is the UT time and the $y$-axis is the deflection angle $\Delta \theta$. The vertical lines in (a), (b), (c), and (d) are the centers of the corresponding current sheets.

background magnetic fields of the two adjacent flux tubes. The deflection angles are about $80^{\circ}$ for case (a) and (b); $100^{\circ}$ for case (c) and $65^{\circ}$ for case (d).

Figure 3 is a sketch to show the behaviors of $\theta(\zeta, t)$ and $\tau$ as a spacecraft crosses a current sheet. Two adjacent flux tubes and a current sheet in between are shown. The blue arrows in the figure display the orientation of the magnetic field in the flux tubes and within the current sheet. In order to simplify the discussion, without losing generality, we assume the magnetic fields $\boldsymbol{B}$ are upright or downright in the two flux tubes. We also assume $\boldsymbol{B}$ in the current sheet linearly rotate from the upright to the downright direction. We assume the first flux tube is from the left side of Fig. 3 to the red line $B$; the second flux tube is from the red line $B^{\prime}$ to the right side of Fig. 3; and the area between lines $B$ and $B^{\prime}$ is the current sheet. According to Eq. (5),

$\theta\left(\zeta, t_{D}\right)=\cos ^{-1}\left(\hat{b}\left(t_{0}\right) \cdot \hat{b}\left(t_{0}^{\prime}\right)\right)$

where $t_{0}=t_{D}-\zeta / 2$ and $t_{0}^{\prime}=t_{D}+\zeta / 2$, is obtained as the angle between the $\boldsymbol{B}$ vectors at $t_{0}$ of the flux tube 1 and $t_{0}^{\prime}$ which is the far left of the current sheet. The time separation between those two vectors is $\zeta$. From the figure, $\theta(\zeta, t)$ starts to increase at time $t_{D}$. Once the $\Delta \theta$ corresponds to two unit vectors $\hat{b}\left(t_{2}\right)$ and $\hat{b}\left(t_{2}^{\prime}\right)$ that reside in adjacent flux tube I and II where $t_{2}=t_{C}-\zeta / 2$ and $t_{2}^{\prime}=t_{C}+\zeta / 2$, it reaches its maximum value:

$\theta\left(\zeta, t_{C}\right)=\cos ^{-1}\left(\hat{b}\left(t_{2}\right) \cdot \hat{b}\left(t_{2}^{\prime}\right)\right)$.

$\theta(\zeta, t)$ keeps the maximum value when $t_{C} \leq t \leq t_{C}^{\prime}$. After $t_{C}^{\prime}, \theta(\zeta, t)$ begins to decrease because the $\Delta \theta$ is calculated between the $\boldsymbol{B}$ vectors at $t_{4}$ and $t_{4}^{\prime}$, which are at the edge of the flux tube I, and in the flux tube II, respectively. When $t=t_{D}^{\prime}, \theta(\zeta, t)$ drops back to its minimum value since both $t_{4}=t_{D}-\zeta / 2$ and $t_{4}^{\prime}=t_{D}+\zeta / 2$ in the flux tube II. Note $\theta(\zeta, t)$ has a shape of isosceles trapezoid. The center of the isosceles trapezoid, marked by the straight line A, is the center of the current sheet. Its location is independent of the value of $\zeta$. In contrast, the lengths of the top and the bottom side of the isosceles trapezoid are $\zeta$-dependent. We use these properties to search for the current sheets. In implementing the searching procedure, five different $\zeta$ values, $20 \mathrm{~s}$, $30 \mathrm{~s}, 40 \mathrm{~s} 60 \mathrm{~s}$, and $80 \mathrm{~s}$ are used. Three pairs of $(\zeta, 2 \zeta)$ : $(20 \mathrm{~s}, 40 \mathrm{~s}),(30 \mathrm{~s}, 60 \mathrm{~s})$, and $(40 \mathrm{~s}, 80 \mathrm{~s})$ are formed. Out of these three pairs, if the relationship of $\tau(\theta, 2 \zeta) \sim 2 \tau(\theta, \zeta)$ is 


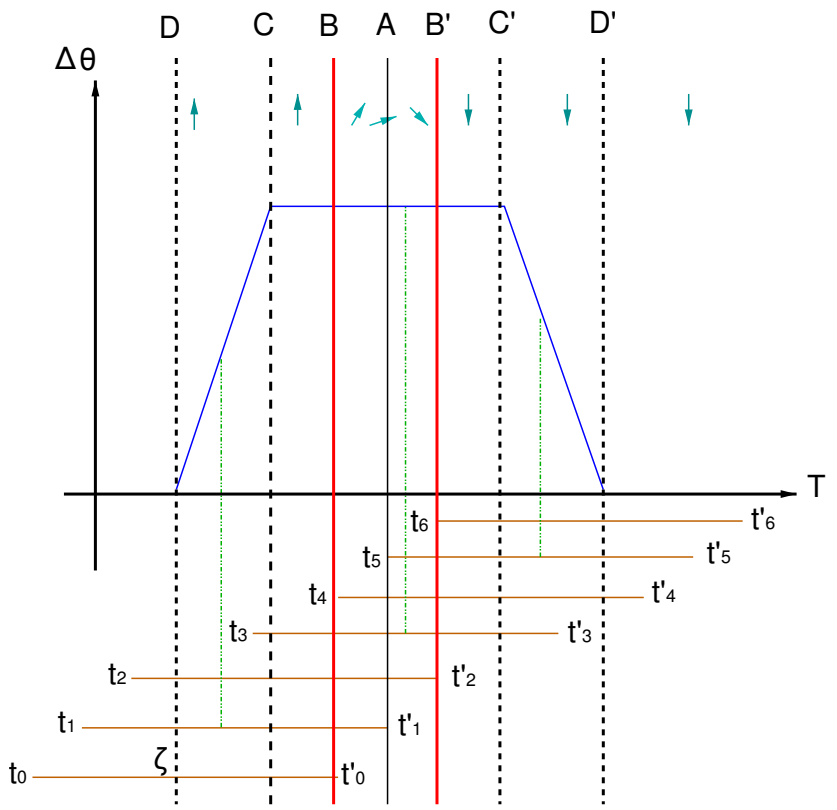

Fig. 3. Cartoon showing the procedure to calculate $\Delta \theta(\zeta, t)$. $\Delta \theta(\zeta, t)$ is plotted in $\Delta \theta-t$ plane. The y-axis is $\Delta \theta(\zeta, t)$ in degree; the $\mathrm{x}$-axis is UT time. The green arrows are the orientation of $\boldsymbol{B}$ vectors. The region from the left side to the red line $B$ is the flux tube 1, which has upright $\boldsymbol{B}$ vector; the region from red line $B^{\prime}$ to the right side is the flux tube 2, which has downright $\boldsymbol{B}$ vector; the region between red lines $B$ and $B^{\prime}$ is the current sheet, in which $\boldsymbol{B}$ vector is changed gradually. The black vertical line $A$ is the center of current sheet.

satisfied more than once, a potential current sheet is registered by the searching code. The code then calculates the angle $\Delta \theta$ using the average from $t_{C}<t<t_{C}^{\prime}$ for this current sheet. From Fig. $3, t_{B}=t_{D}+\zeta / 2, t_{D}^{\prime}=t_{B}^{\prime}+\zeta / 2$ and $t_{B}^{\prime}-t_{B}=d$. So the separation between the two bottom vortices of isosceles trapezoid is $t_{D}^{\prime}-t_{D}=\zeta+d$. Similarly, due to $t_{B}^{\prime}=t_{C}+\zeta / 2$ and $t_{C}^{\prime}=t_{B}+\zeta / 2$, the length of the top side of the isosceles trapezoid is $\zeta-d$. Thus, $d$ can be determined if the top vortices of isosceles trapezoid is known.

\subsection{Determining the thickness and the deflection angle of a current sheet}

We now discuss in detail how $\Delta \theta$ and $d$ are obtained. Although the center of a current sheet can be rather nicely identified as illustrated in Fig. 3, an accurate value of the deflection angle $\Delta \theta$ and the width $d$ of the current sheet is hard to obtain due to the uncertainties in identifying the vortices of the isosceles trapezoid. To better decide the width of a current sheet, a separate routine is developed. Figure 4 shows the actual procedure of this code. The left panel of Fig. 4 shows how the shape of $\Delta \theta(\zeta, t)$ varies with $\zeta$. Consider again the ideal model of a current sheet which is shown in Fig. 3. In the isosceles trapezoid shown in the bottom of the left side of Fig. 4, th top side of the isosceles trapezoid has a length $\zeta-d$ and the bottom side of isosceles trapezoid has a length $\zeta+d$. If we further gradually decrease $\zeta$, then upon reaching a critical $\zeta_{0}$ the top side will disappear and the isosceles trapezoid becomes an isosceles triangle. We have now, $\zeta_{0}-d=0$. Therefore the width of current sheets can be determined as the critical $\zeta_{0}$. Note, If the $\zeta$ is further decreased below $\zeta_{0}$, the maximum value of $\theta(\zeta, t)$ will begin to decrease. This is because that if the $\zeta$ is less than the width of a current sheet, then $\theta(\zeta, t)$ is not the angle between the two $\boldsymbol{B}$ vectors that reside in different flux tubes. Instead, it becomes the angle between two magnetic field vectors within the current sheet. This fact makes the identification of $\zeta_{0}$ more robust.

In the code, we first calculate $\Delta \theta\left(\zeta, t_{0}\right)$ for $\zeta=1$ to $\zeta=$ $300 \mathrm{~s}$. (300 is chosen as it is larger than the width of all current sheets identified in this work) and then search for a plateau region of $\Delta \theta\left(\zeta, t_{0}\right)$. The width of the current sheet is then decided to be $\zeta_{0}$, the $\zeta$ value for the starting point of the plateau region; and the deflection angle $\Delta \theta$ of the current sheet is decided to be $\Delta \theta\left(\zeta_{0}, t_{0}\right)$

An example of deciding $d$ and $\Delta \theta$ for the event shown in the panel (c) of Fig. 2 is shown in the right panel of Fig. 4. Using the method described as above, the thickness of this current sheet is obtained as $40 \mathrm{~s}$.

\section{Data analysis and results}

Applying the automatic searching code to the data selection periods A and B, which are shown in Fig. 1, 28214 current sheets are found in about 3.2 years. In Fig. 5, from the top panel to the bottom panel, $B_{\mathrm{t}}, V_{\mathrm{SW}}$, and current sheet density are shown as a function of time, respectively. The time span shown in the left and right sides of the figure correspond to the region A and B displayed in Fig. 1. The large periodic changes of $B_{\mathrm{t}}$ and $V_{\mathrm{SW}}$ are due to the rotation of the Sun. The 3rd panel shows the number of current sheets per day. From the figure we see that the current sheet occurrences rate can vary from tens events per day down to several per day and occasionally reach even zero event per day. The variation of the current sheet occurrence rate implies that current sheets are clustered. Part of this clustering is related to the solar wind speed. From Fig. 5, we can see clearly that there are more current sheets within fast streams than within slow streams. This implies that fast wind is more intermittent than slow wind, which is different from earlier finding (Marsch and Liu, 1993) which suggested that slow wind is more intermittent than fast wind. However, While solar wind speed has a strong correlation with the current sheet occurrence rate, it can not be the only reason for the clustering as one can see from Fig. 5 (for example, between July 1997 to the end of 1997) that even within slow solar wind the current sheet occurrence still have large variations. A clustering effect is what one would expect to find if the data 

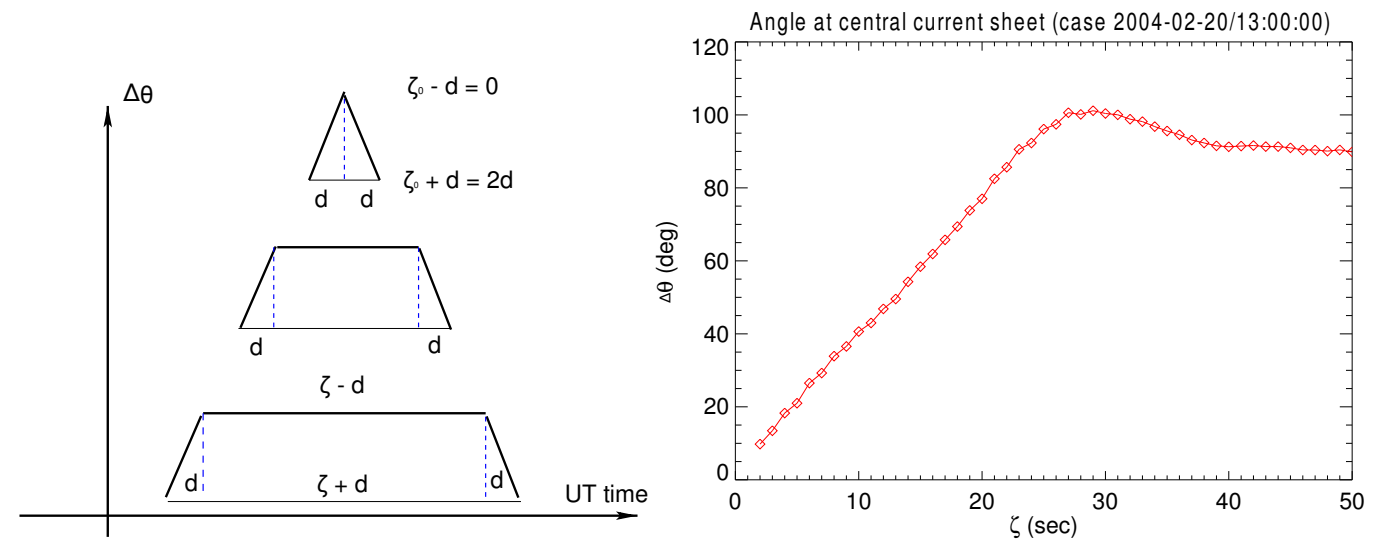

Fig. 4. Figure showing how $d$ and $\Delta \theta$ are obtained. The left panel shows how the shape of $\Delta \theta(\zeta, t)$ varies with $\zeta$. $\Delta \theta(\zeta, t) \mathrm{s}$ for three $\zeta \mathrm{s}$ are shown. The bottom part corresponds to the largest $\zeta$, which is taken to be larger than the width of the current sheet, $d$. The shape of $\Delta \theta$ as a function of time is an isosceles trapezoid. The top side has a length of $\zeta-d$ and the bottom side has a length of $\zeta+d$. As $\zeta$ decreases, this isosceles trapezoid gets compressed, until a critical value of $\zeta_{0}=d$ is reached, in which case, the isosceles trapezoid becomes an isosceles triangle. The right panel is an actual case showing the deflection angle $\Delta \theta\left(\zeta, t_{0}\right)$ as a function of $\zeta$. Here $t_{0}$ is at the center of current sheet. As $\zeta$ increases from zero, the deflection increases linearly at the beginning, reflecting the fact that the current sheet has a finite width and the direction of magnetic field within the current sheet changes gradually inside the current sheet. At large $\zeta \mathrm{s}, \Delta \theta$ reaches a plateau, signaling the fact that $\zeta$ is now bigger than $d$.

is intermittent. Indeed, Greco et al. (2009), who used both a numerical simulation and actual solar wind data analysis showed that the distribution of waiting time between discontinuities is not Poisson but has characteristics of clustering.

\subsection{Deflection angle $\Delta \theta$ between adjacent flux tubes}

We now discuss the deflection angle across the current sheets. Our code automatically identify the deflection angle. By definition, the deflection angle should be larger than the background value of $\cos ^{-1}(\hat{B}(t) \cdot \hat{B}(t+\delta))$ where $\delta$ is the time resolution of the magnetic field data. If the direction of the background magnetic field change rather rapidly and $\cos ^{-1}(\hat{B}(t) \cdot \hat{B}(t+\delta))$ is say, about 10 degrees, then our method will not resolve current sheets which has a deflection angle smaller than 10 degrees.

The distributions of the deflection angle $\Delta \theta$ across current sheets are shown in Fig. 6. One important feature from Fig. 6 is that there is a break point in the distribution of the deflection angle $\Delta \theta$ around $\Delta \theta=72^{\circ}$. This break point separates the distribution of $\Delta \theta$ to two populations. The population with $\theta>72^{\circ}$ can be fitted by an exponential decay with a functional form of

$\mathrm{PDF} \sim A_{1} e^{-\Delta \theta / 30.0^{\circ}}$.

The fit is shown as the red dashed line. The population with $\theta<72^{\circ}$ can be also fitted by an exponential decay with a functional form of

$\mathrm{PDF} \sim A_{2} e^{-\Delta \theta / 18.6^{\circ}}$.

The fit is shown as the blue dashed line and is done for $25^{\circ}<\Delta \theta<72^{\circ}$. Here $A_{1}$ and $A_{2}$ are constants. The fact that the distribution shows a two population suggests that the origin of these current sheets for these two populations may be different. Indeed, such a two population of the distribution of $\Delta \theta$ have been first discussed by Bruno et al. (2004) where the authors suggested that the first population with small deflection angle may be caused by the intrinsic turbulence, and the second population with large deflection angle may be caused by relic structures originated from the surface of the Sun. Later Borovsky (2008) used ACE magnetic field data to examine the distribution of the deflection angle between two magnetic fields with a fixed $120 \mathrm{~s}$.

Our result is consistent with the study of Bruno et al. (2004) and Borovsky (2008). The exponential decay constants in our study, however, are smaller than that obtained in (Borovsky, 2008) for both populations.

\subsection{Width of current sheets}

We next discuss the distribution of current sheet width in Fig. 7. Note, the real width of a current sheet depends on how the spacecraft cross the current sheet, i.e. the relative velocity between the current sheet and the spacecraft. Since this can not be obtained from a single spacecraft observation, we denote here the width of a current sheet by the crossing time duration $\tau$.

Since from Fig. 6 we obtain two population of the current sheet, we therefore examine the current sheet width for these two population separately. Near $\theta=80^{\circ}$ the two population are not clearly separated. So for the first population we require,

$$
10^{\circ}<\theta^{\mathrm{I}}<20^{\circ}
$$



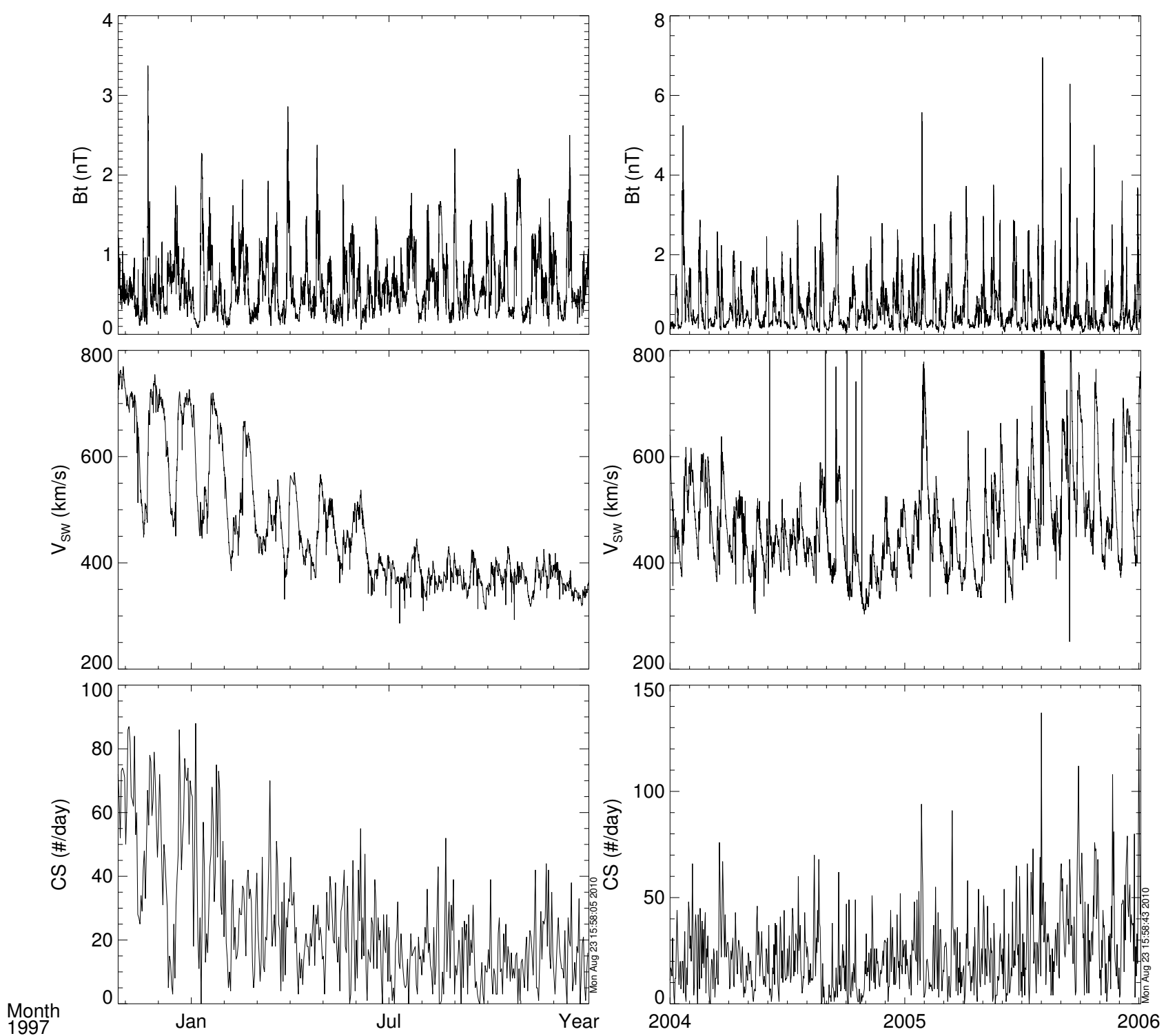

Fig. 5. $B_{\mathrm{t}}, V_{\mathrm{SW}}$, and current sheet density are plotted as functions of UT time. The time period in the left side is from 1996-300 to 1997-365. The time period in the right side is from 2004-1 to 2006-3. The current sheet density is calculated as the number of current sheet occurrences per day.

and for the second population, we require

$100^{\circ}<\theta^{\mathrm{II}}<180^{\circ}$

In Fig. 7 we plot the distribution of current sheet width for these two populations. The $\mathrm{x}$-axis is the current sheet width $\tau$ and the y-axis is the probability density $P(\tau) . P(\tau)$ represents the probability of finding a current with a width between $\tau \mathrm{s}$ and $\tau+1 \mathrm{~s}$. If we define

$F(\tau)=\int_{\theta_{<}}^{\theta_{>}} f(\Delta \theta, \tau) \mathrm{d} \Delta \theta$ the $P(\tau)$ can be written as,

$$
P(\tau)=\frac{F(\tau)}{\int F(\tau) d \tau .}
$$

In Eq. (12), $\theta_{<}=10^{\circ}$ and $\theta_{>}=20^{\circ}$ for the Population I (the small angle population) and $\theta_{<}=100^{\circ}$ and $\theta_{>}=180^{\circ}$ for the Population II (the large angle population). The black curve in Fig. 7 is for Population I and the red curve is for Population II. From the figure we can see that there is a clear difference between these two populations. The black curve is very narrowly peaked. It has a peak value around $13 \mathrm{~s}$. In comparison, the red curve is more spreading out. There is no 


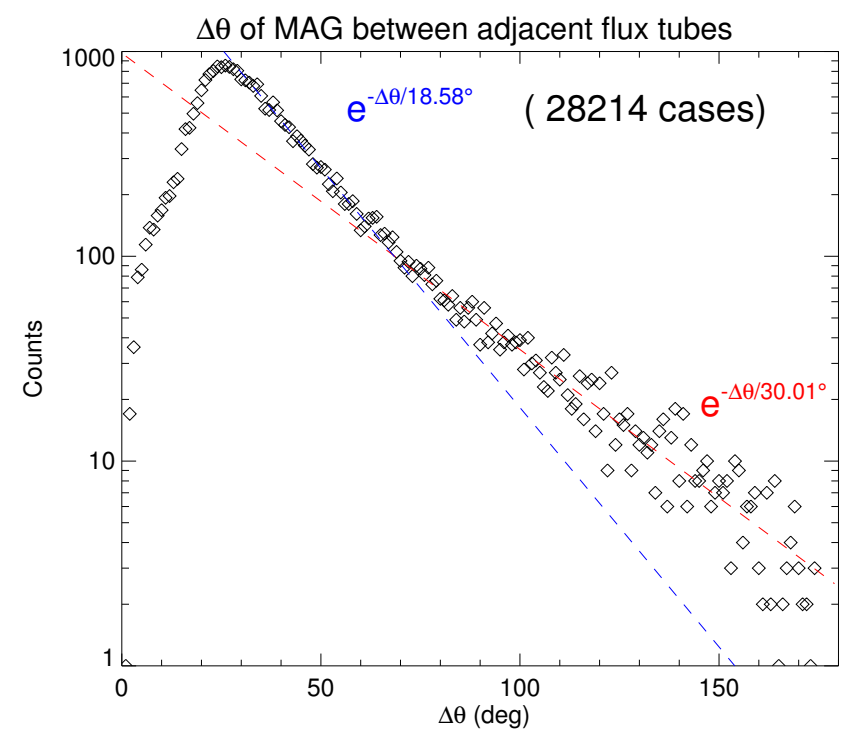

Fig. 6. Angle between the magnetic field of adjacent flux tube. The red dashed line is the fitting curve by using exponential function $A_{1} e^{-\Delta \theta / 30.01^{\circ}}, A_{1}$ is a constant. The blue dashed line is the fitting curve by using exponential function $A_{2} e^{-\Delta \theta / 18.58^{\circ}}, A_{2}$ is a constant.

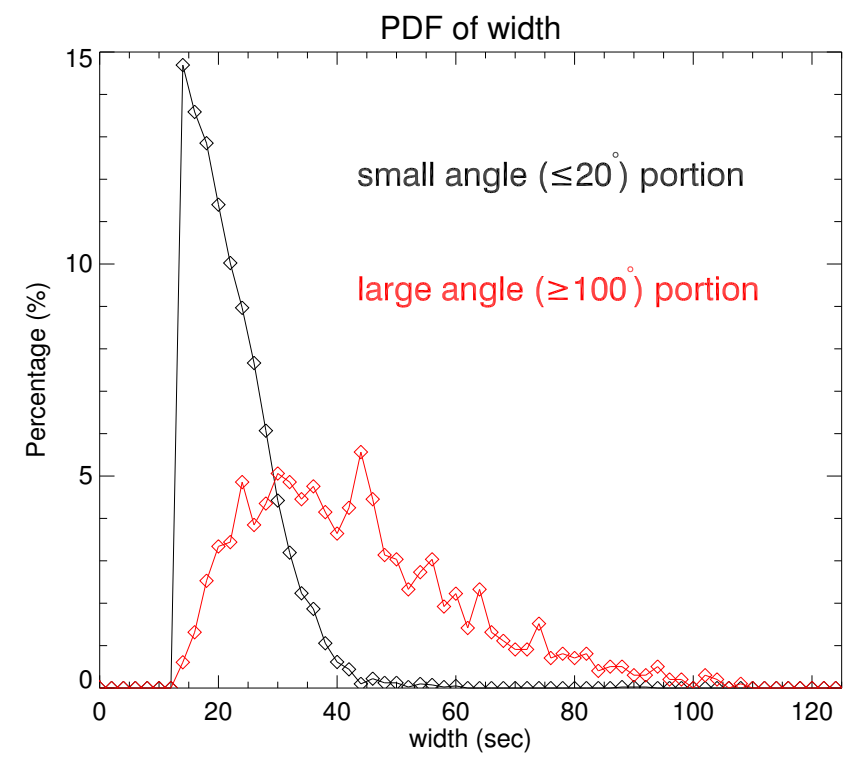

Fig. 7. Width of current sheet of all 28214 cases. The y-axis is the normalized number of occurrences of current sheet in log scale; the $\mathrm{x}$-axis is width of current sheet, unit is second. The black curve is the potion with $\Delta \theta<20^{\circ}$; the red curve is the potion with $\Delta \theta>$ $100^{\circ}$.

clear peak for the red curve; instead the percentage is approximately a constant between $20 \mathrm{~s}$ and $45 \mathrm{~s}$ for the red curve. It then decreases gradually and extends to beyond $100 \mathrm{~s}$, therefore having a longer tail at large width. This is consistent with the two population scenario as we expect the width for

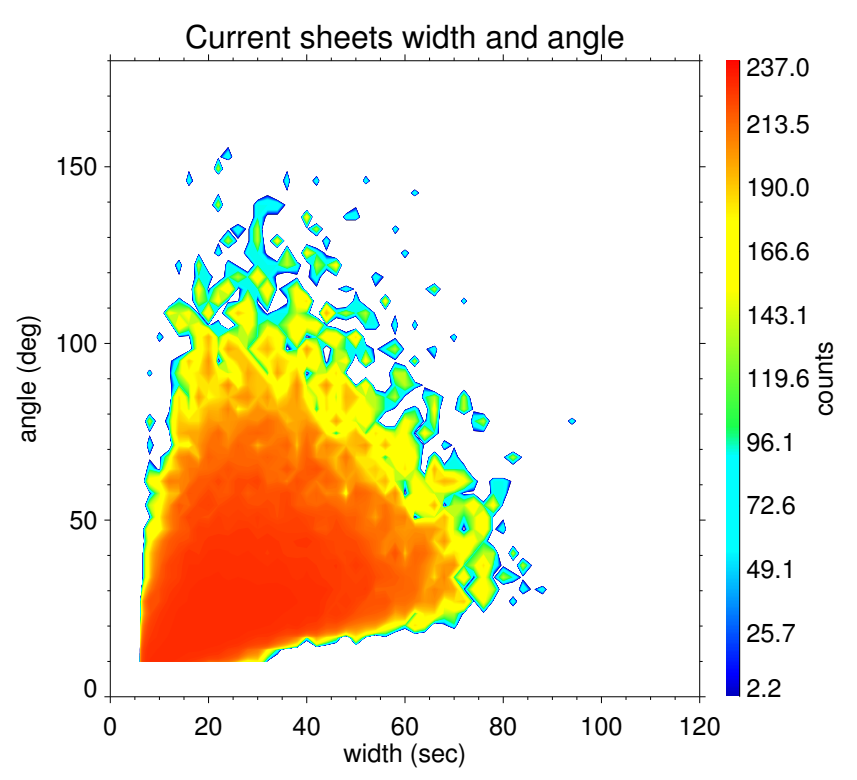

Fig. 8. The correlation of the width of current sheets and angle between the magnetic field of adjacent flux tube. In the left part, the $\mathrm{y}$-axis is the $\Delta \theta(\mathrm{deg})$, and the $\mathrm{x}$-axis is the width (s). The colors in the left part are the counts of current sheet occurrences. The pattern is smoothed by the interpolation.

the large angle population is somewhat larger than that of the small angle population. In a related study, Tsurutani et al. (2009) studied magnetic decreases in the solar wind using Ulysses observations and found these structures have a temporal thickness ranging from $1 \mathrm{~s}$ to beyond $100 \mathrm{~s}$.

Figure 8 is a 2-D plot of the probability density $f(\Delta \theta, \tau)$ in the $\Delta \theta-\tau$ plane. The color represents the number of current sheets per degree per second. Red indicates more current sheets and blue indicate fewer current sheets. From the figure we see that $f(\Delta \theta, \tau)$ has a triangle-like shape with most of the current sheet located at the lower and left part of the triangle indicating that current sheets with small deflection angles and small width are the most popular. As $\theta$ increases, we see the red color in the left edge gradually moves to the right. This can be understood from the fact that current sheets with larger deflection angles tend to be wider.

\subsection{Waiting time analysis}

For a time series data, we can perform waiting time analysis. A waiting time analysis is particularly useful in understanding the temporal behavior of intermittent events. Indeed, if a time series data is intermittent, we expect the waiting time analysis to be non-Poisson, therefore waiting time analysis can be used to reveal the statistical property of the intermittency. Previous work on using the waiting time analysis to investigate the solar wind MHD turbulence intermittency can be found, e.g. in Lepreti et al. (2001); Carbone et al. (2006); Greco et al. (2009). 


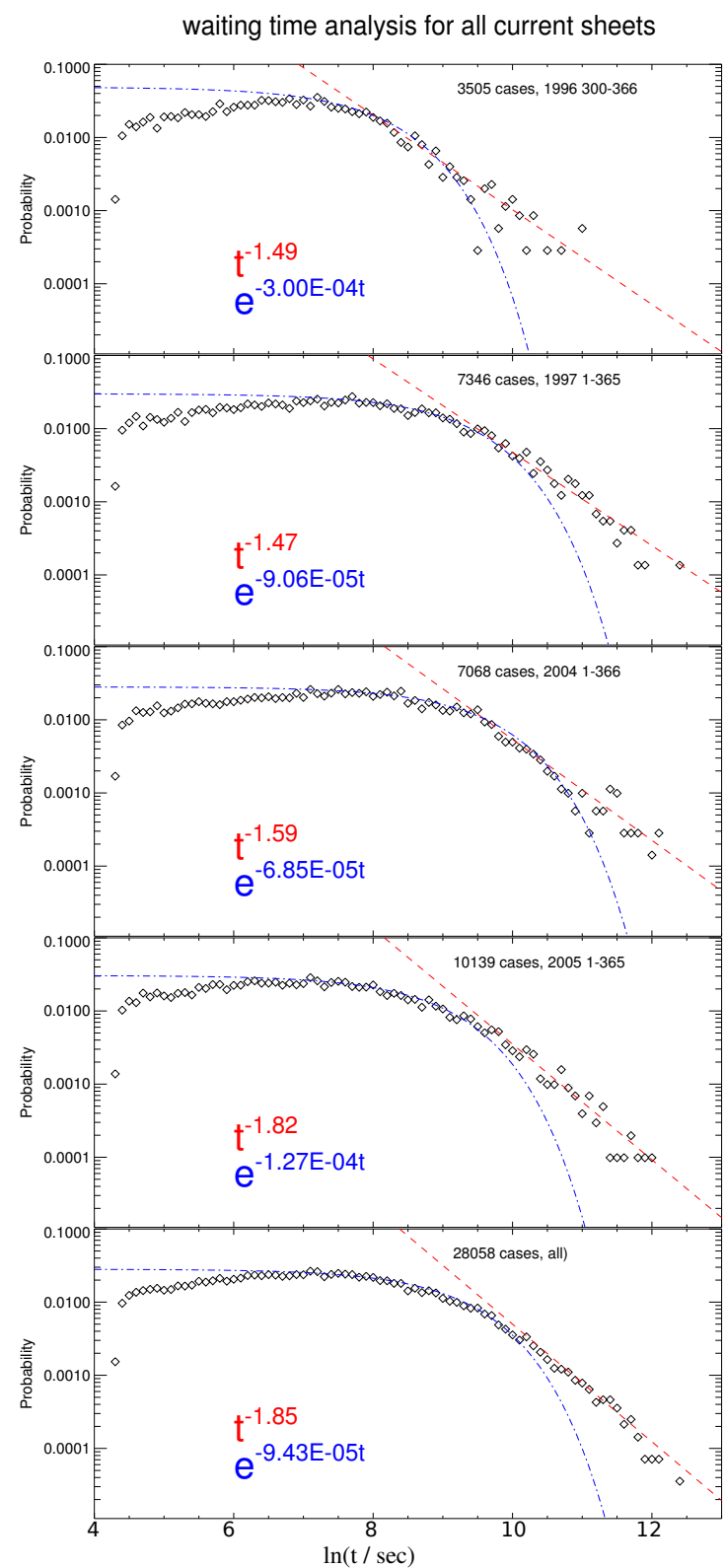

waiting time analysis for large deflection angle current sheets

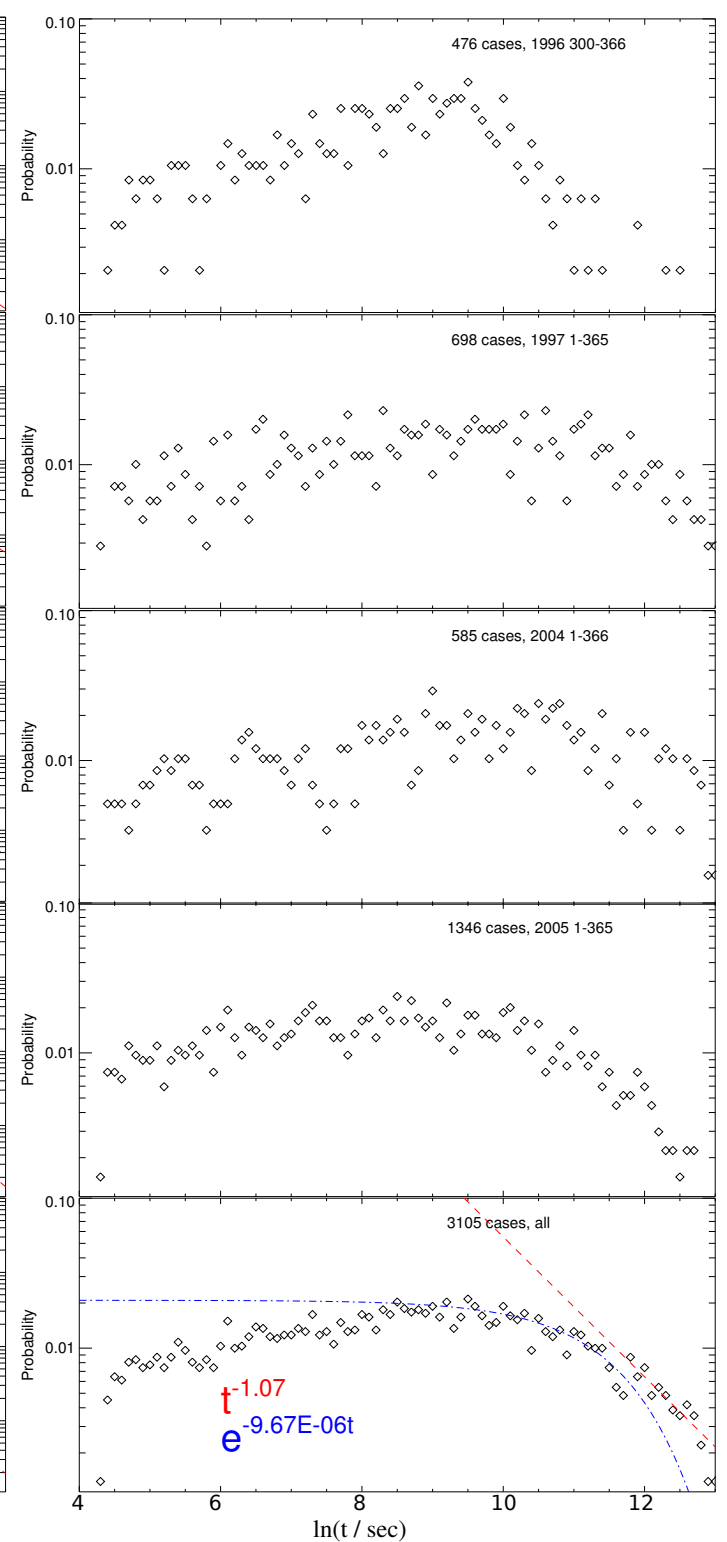

Fig. 9. Waiting time analysis in different years. The left side is the waiting time analysis on the current sheets with all deflection angle; the right side is the waiting time analysis on the current sheets with only large deflection angle $\left(\Delta \theta \geq 72^{\circ}\right)$. From top to the bottom, the first panel is the waiting time analysis from DOY 300 to DOY 366 in 1996; the 2nd, 3rd, and 4th panels are the waiting time analysis in 1997, 2004, and 2005, respectively. The bottom panel is the waiting time analysis for all current sheet cases. The y-axis is the logarithm of the probability of current sheet occurrences and the $\mathrm{x}$-axis is the logarithm of waiting time, $\ln (t / \mathrm{sec})$.

We now perform the waiting time analysis for our current sheets. If these current sheets are the boundaries of flux tube, then the waiting time between the current sheets can be used as a proxy of the size of the flux tube. If these current sheets are natural structures of the solar wind MHD turbulence (Zhou et al., 2004), this waiting time analysis would reveal their clustering tendency. The statistical distribution of waiting time is shown in Fig. 9. From the top to the bottom, the waiting time analysis is done in each individ- ual year and all years, respectively. One exception is year 1996 where the waiting time analysis is based on the observation from DOY 300 to DOY 366. The distributions in the left side of Fig. 9 are the waiting time analysis for all current sheet events. In comparison, the distributions in the right side of Fig. 9 are the waiting time analysis for current sheet events with large deflection angle $\left(\Delta \theta \geq 72^{\circ}\right)$. The $\mathrm{x}$-axis is the logarithm of time, $\ln (t / \mathrm{s})$ and the $\mathrm{y}$-axis is logarithm of the probability density. The $\mathrm{x}$-axis shown 
in the figure starts at $\ln (t / \mathrm{sec})=4$ which corresponds to a time $t=55 \mathrm{sec}$ and ends at $\ln (t / \mathrm{sec})=13$ which corresponds to a time $t=4.4 \times 10^{5} \mathrm{sec} \sim 123 \mathrm{~h}$. The increase is $\ln (t / \mathrm{sec})=0.5$ per tick. Following Greco et al. (2009), we also fit the distributions by an exponential decay at small times and a power law at large times. The blue curves are the exponential decay and the red curves are the power laws. For the case of all current sheets on the left panel, one can see that the exponential decay fittings agree well with the observation at small times but underestimate the observation at large times where the red power law fittings agree better. For the large deflection angle plots on the right, the statistics are poor and the fitting is done only for the all-year plot. Again, a power law like tail can be seen at large times. Comparing to the all angle plots on the left, however, this power law is harder.

The most probable waiting time $\lambda$ s are listed in Table 1. For the large angle population we see that the most probably waiting times are $\sim 3.5 \mathrm{~h}$. As a first proxy, this can be taken as the average size of the flux tubes. In comparison, the most probable waiting times for all population (which is mainly due to the small angle population) are $\sim 0.4 \mathrm{~h}$, considerably smaller.

In the work of Greco et al. (2009), Greco et al. used both numerical simulation and solar wind data analysis to examine the waiting times for discontinuities in the solar wind. Greco et al. (2009) found that the waiting time density is a power law with a break. A power law distribution suggested that the discontinuities in the solar wind are clustered. In our case, for the all angle analysis (the left panels), the distributions behave like exponential decays at small $t$ s and power laws when $t>\lambda$. This is in agreement with (Greco et al., 2009). For the large angle analysis, the statistics for individual years are poor. For the all-period analysis, the distribution at large $t \mathrm{~s}$ is consistent with a power law, but is harder than that for the all angle analysis. This also supports the conjecture that the large angle population may originate via a different mechanism from that of the small angle population.

\section{Conclusions}

In this paper, we extended the work of $\mathrm{Li}(2007,2008)$ and developed an automatic data analysis procedure to identify current sheets in the solar wind. The procedure obtains the location and the width (thickness) of individual current sheets and the deflection angle of the magnetic field across the current sheet. Applying our method to 3-year worth magnetic field data from the Ulysses mission, we have identified 28214 current sheets during solar minimum period and at low heliospheric latitude. The distribution of the width of these current sheets and the distribution of the deflection angle are shown in Figs. 7 and 6. We also perform the waiting time analysis on these current sheets. The distribution of the waiting time of these current sheets is shown in Fig. 9.
Table 1. The most intermittent scales.

\begin{tabular}{lll}
\hline Year & $\begin{array}{l}\lambda_{1} \\
\text { (cases with all angles })\end{array}$ & $\begin{array}{l}\lambda_{2} \\
(\text { cases with large angles })\end{array}$ \\
\hline 1996 & $e^{7.2} \sim 1339.4 \mathrm{~s} \sim 0.4 \mathrm{~h}$ & $e^{9.5} \sim 13359.7 \mathrm{~s} \sim 3.7 \mathrm{~h}$ \\
1997 & $e^{7.7} \sim 2208.3 \mathrm{~s} \sim 0.6 \mathrm{~h}$ & $e^{8.3} \sim 4023.9 \mathrm{~s} \sim 1.1 \mathrm{~h}$ \\
2004 & $e^{7.1} \sim 1212.0 \mathrm{~s} \sim 0.3 \mathrm{~h}$ & $e^{9.0} \sim 8103.1 \mathrm{~s} \sim 2.3 \mathrm{~h}$ \\
2005 & $e^{7.1} \sim 1212.0 \mathrm{~s} \sim 0.3 \mathrm{~h}$ & $e^{8.5} \sim 4914.8 \mathrm{~s} \sim 1.4 \mathrm{~h}$ \\
All & $e^{7.1} \sim 1212.0 \mathrm{~s} \sim 0.3 \mathrm{~h}$ & $e^{9.5} \sim 13359.7 \mathrm{~s} \sim 3.7 \mathrm{~h}$ \\
\hline
\end{tabular}

In an earlier paper, Bruno et al. (2004) first suggested that there are two populations of current sheets in the solar wind and the second population could have been related to flux-tube boundaries. Our analysis agrees with Bruno et al. (2004)'s finding. We also find two populations of current sheets in the solar wind. These two populations differ in their distributions of the deflection angle across the current sheet and the width of the current sheet. While the origin of these current sheets is still under debate, the suggestion by Bruno et al. (2004) that the large angle population could represent the boundaries of flux tubes is certainly a very attractive one. Besides large angle current sheets, we also find current sheets that have small deflection angles. These current sheets perhaps are dynamically developed in the solar wind MHD turbulence as shown in Zhou et al. (2004) where intermittent structures can naturally develop in MHD turbulence from an initially homogeneous state. These small angle current sheets therefore represent the intrinsic intermittency of the solar wind MHD turbulence.

In a recent review, Neugebauer and Giacalone (2010), noting the work of Vasquez et al. (2007) and Borovsky (2008) who advocated that the in situ generation of discontinuities by Alfvénic turbulence (Vasquez et al., 2007) and the discontinuities with large rotation angles being the boundaries of flux tubes originated at the Sun (Borovsky, 2008), respectively, concluded that both the Sun and turbulence are important sources of interplanetary discontinuities. They further argued that in the slow solar wind, in-situ generation by phase-steepened edges of non-linear Alfvén waves (Tsurutani et al., 2005a,b) may be a manifestation of exhaust fan reconnection.

Regardless of their origins, our method presented here can be used to identify individual current sheets of both population. It also allows one to obtain various properties of these current sheets, such as the distributions of the thickness and the deflection angle of the current sheets and the distribution of the waiting time between adjacent current sheets. As such, our method provides a working basis for studying solar wind MHD turbulence intermittency. 
Acknowledgements. We thank the Ulysses VHM/FGM team for providing the magnetic field data used in this investigation. This work is supported in part by NSF ATM-0847719 (CAREER) and NASA NNX07AL52A (EpSCoR) as well as an ORAU Ralph E. Power Junior Faculty Enhancement Award. We also thank the two referees for very helpful comments.

Topical Editor R. Forsyth thanks two anonymous referees for their help in evaluating this paper.

\section{References}

Balogh, A., Beek, T. J., Forsyth, R. J., Hedgecock, P. C., Marquedant, R. J., Smith, E. J., Southwood, D. J., and Tsurutani, B. T.: The magnetic field investigation on the Ulysses mission: Instrumentation and preliminary scientific results, Astron. Astrophys. Suppl., 92, 221-236, 1992.

Bartley, W. C., Bukata, R. P., McCracke, K. G., and Rao, U. R.: Anisotropic cosmic radiation fluxes of solar origin, J. Geophys. Res., 71, 3297-3304, 1966.

Biskamp, D.: Nonlinear Magnetohydrodynamics, Cambridge University Press, 1993.

Borovsky, J. E.: Flux tube texture of the solar wind: Strands of the magnetic carpet at 1 AU?, J. Geophys. Res-Space Phys., 113, A08110, doi:10.1029/2007JA012684, 2008.

Bruno, R. and Carbone, V.: The Solar Wind as a Turbulence Laboratory, Living Reviews in Solar Physics, 2, http://www. livingreviews.org/lrsp-2005-4, 2005.

Bruno, R., Bavassano, B., Pietropaolo, E., Carbone, V., and Veltri, P.: Effects of intermittency on interplanetary velocity and magnetic field fluctuations anisotropy, Geophys. Res. Lett., 26, 3185-3188, 1999.

Bruno, R., Carbone, V., Veltri, P., Pietropaolo, E., and Bavassano, B.: Identifying intermittency events in the solar wind, Planet Space Sci., 49, 1201-1210, 2001.

Bruno, R., Carbone, V., Sorriso-Valvo, L., and Bavassano, B.: Radial evolution of solar wind intermittency in the inner heliosphere, J. Geophys. Res.-Space Phys., 108, 1130, doi:10.1029/ 2002JA009615, 2003.

Bruno, R., Carbone, V., Primavera, L., Malara, F., Sorriso-Valvo, L., Bavassano, B., and Veltri, P.: On the probability distribution function of small-scale interplanetary magnetic field fluctuations, Ann. Geophys., 22, 3751-3769, doi:10.5194/angeo-223751-2004, 2004.

Burlaga, L. F.: Multifractal structure of the interplanetary magneticfield - VOYAGER 2 observations near 25 AU, 1987-1988, Geophys. Res. Lett., 18, 69-72, 1991a.

Burlaga, L. F.: Multifractal structure of speed fluctuations in recurrent streams at $1-\mathrm{AU}$ and near 6-AU, Geophys. Res. Lett., 18, 1651-1654, 1991b.

Burlaga, L. F.: Intermittent turbulence in the solar-wind, J. Geophys. Res-Space Phys., 96, 5847-5851, 1991c.

Carbone, V., Bruno, R., and Veltri, P.: Scaling laws in the Solar Wind turbulence, Springer-Verlag, doi:10.1007/BFb0102411, http://www.springerlink.com/content/R553714R3U577101, 1995a.

Carbone, V., Veltri, P., and Bruno, R.: Experimental-evidence for differences in the extended self-similarity scaling laws between fluid and magnetohydrodynamic turbulent flows, Phys. Rev. Lett., 75, 3110-3113, 1995b.
Carbone, V., Sorriso-Valvo, L., Vecchio, A., Lepreti, F., Veltri, P., Harabaglia, P., and Guerra, I.: Clustering of polarity reversals of the geomagnetic field, Phys. Rev. Lett., 96, 128501, doi:10.1103/ PhysRevLett.96.128501, 2006.

Chang, T.: Self-organized criticality, multi-fractal spectra, sporadic localized reconnections and intermittent turbulence in the magnetotail, Phys. Plasmas, 6, 4137-4145, 1999.

Chang, T., Tam, S., and Wu, C.: Complexity induced anisotropic bimodal intermittent turbulence in space plasmas, Phys. Plasmas, 11, 1287-1299, doi:10.1063/1.1667496, 2004.

Goldstein, M. L., Roberts, D. A., and Matthaeus, W. H.: Magnetohydrodynamic turbulence in the solar-wind, Annu. Rev. Astron. Astrophys., 33, 283-325, 1995.

Greco, A., Matthaeus, W. H., Servidio, S., and Dmitruk, P.: Waiting-time distributions of magnetic discontinuities: Clustering or Poisson process?, Phys. Rev. E, 80, 046401, doi:10.1103/ PhysRevE.80.046401, 2009.

Horbury, T., Balogh, A., Forsyth, R., and Smith, E.: Magnetic field signatures of unevolved turbulence in solar polar flows, J. Geophys. Res-Space Phys., 101, 405-413, 1996.

Horbury, T., Balogh, A., Forsyth, R., and Smith, E.: Ulysses observations of intermittent heliospheric turbulence, Heliosphere at solar minimum and beyond, 19, 847-850, 1997.

Iroshnikov, P. S.: Turbulence of a Conducting Fluid in a Strong Magnetic Field, Soviet Astronomy, 7, 566-571, 1964.

Kolmogorov, A.: The local structure of turbulence in incompressible viscous fluid for very large Reynolds numbers, C. R. Acad. Sci. URSS, 30, 301-305, 1941.

Kraichnan, R. H.: Inertial-range spectrum of hydromagnetic turbulence, Phys. Fluids, 8, 1385-1387, 1965.

Lepreti, F., Carbone, V., and Veltri, P.: Solar flare waiting time distribution: Varying-rate Poisson or Levy function?, Astrophys J., 555, L133-L136, 2001.

Li, G.: Flux tubes in the fast and slow solar wind, AIP Conference Proceedings, 932, 26-31, doi:10.1063/1.2778941, http://link.aip. org/link/?APC/932/26/1, 2007.

Li, G.: Identifying current-sheet-like structures in the solar wind, Astrophys. J. Lett., 672, L65-L68, 2008.

Li, G., Lee, E., and Parks, G.: Are there current-sheet-like structures in the Earth's magnetotail as in the solar wind - results and implications from high time resolution magnetic field measurements by Cluster, Ann. Geophys., 26, 1889-1895, doi:10.5194/angeo26-1889-2008, 2008.

Mariani, F., Bavassan.B, Villante, U., and Ness, N.: Variations of occurrence rate of discontinuities in interplanetary magneticfield, J. Geophys. Res., 78, 8011-8022, 1973.

Marsch, E. and Liu, S.: Structure functions and intermittency of velocity fluctuations in the inner solar-wind, Ann. Geophys.Atmos. Hydrospheres Space Sci., 11, 227-238, 1993.

Marsch, E. and Tu, C.: Non-gaussian probability-distributions of solar-wind fluctuations, Ann. Geophys.-Atmos. Hydrospheres Space Sci., 12, 1127-1138, 1994.

Marsch, E. and Tu, C.-Y.: Intermittency, non-Gaussian statistics and fractal scaling of MHD fluctuations in the solar wind, Nonlin. Processes Geophys., 4, 101-124, doi:10.5194/npg-4-101-1997, 1997.

McCracken, K. and Ness, N.: Collimation of cosmic rays by interplanetary magnetic field, J. Geophys. Res., 71, 3315-3318, 1966.

Meneveau, C. and Sreenivasan, K.: Simple multifractal cascade 
model for fully-developed turbulence, Phys. Rev. Lett., 59, 1424-1427, 1987.

Neugebauer, M. and Giacalone, J.: Progress in the study of interplanetary discontinuities, AIP Conf. Proc.,, 1216, 194, 2010.

Ruzmaikin, A., Feynman, J., Goldstein, B., Smith, E., and Balogh, A.: Intermittent turbulence in solar-wind from the south polar hole, J. Geophys. Res-Space Phys., 100, 3395-3403, 1995.

Salem, C., Mangeney, A., Bale, S. D., Veltri, P., and Bruno, R.: Anomalous scaling and the role of intermittency in solar wind MHD turbulence: new insights, AIP Conference Proceedings, 932, 75-82, doi:10.1063/1.2778948, http://link.aip. org/link/?APC/932/75/1, 2007.

Salem, C., Mangeney, A., Bale, S. D., and Veltri, P.: SOLAR WIND MAGNETOHYDRODYNAMICS TURBULENCE: ANOMALOUS SCALING AND ROLE OF INTERMITTENCY, Astrophys. J., 702, 537-553, doi:10.1088/0004-637X/702/1/537, 2009.

Tsurutani, B. T., Guarnieri, F. L., Lakhina, G. S., and Hada, T.: Rapid evolution of magnetic decreases (MDs) and discontinuities in the solar wind: ACE and CLUSTER, Geophys. Res. Lett., 32, L10103, doi:10.1029/2004GL022 151., 2005a.

Tsurutani, B. T., Lakhina, G. S., Pickett, J. S., Guarnieri, F. L., Lin, N., and Goldstein, B. E.: Nonlinear Alfvén waves, discontinuities, proton perpendicular acceleration, and magnetic holes/decreases in interplanetary space and the magnetosphere: intermediate shocks?, Nonlin. Processes Geophys., 12, 321-336, doi:10.5194/npg-12-321-2005, 2005b.

Tsurutani, B. T., Guarnieri, F. L., Echer, E., Lakhina, G. S., and Verkhoglyadova, O. P.: Magnetic decrease formation from $i$ 1 AU to similar to $5 \mathrm{AU}$ : Corotating interaction region reverse shocks (vol 114, A12102, 2009), J. Geophys. Res.-Space Physics, 114, A08105, doi:10.1029/2009JA015018, 2009.
Tu, C.: The damping of interplanetary Alfvenic fluctuations and the heating of the solar-wind, J. Geophys. Res.-Space Phys., 93, 7-20, 1988.

Tu, C. and Marsch, E.: MHD structures, waves and turbulence in the solar-wind - observations and theories, Space Sci. Rev., 73 , 1-210, 1995.

Tu, C., Marsch, E., and Rosenbauer, H.: An extended structurefunction model and its application to the analysis of solar wind intermittency properties, Ann. Geophys.-Atmos. Hydrospheres Space Sci., 14, 270-285, 1996.

Vasquez, B. J., Abramenko, V. I., Haggerty, D. K., and W., S. C.: Numerous small magnetic field discontinuities of Bartels rotation 2286 and the potential role of Alfvenic turbulence,, J. Geophys. Res., 112, A11102, doi:10.1029/2007JA012504, 2007.

Veltri, P. and Mangeney, A.: Scaling laws and intermittent structures in solar wind MHD turbulence, in: American Institute of Physics Conference Series, edited by: Habbal, S. R., Esser, R., Hollweg, J. V., and Isenberg, P. A., vol. 471 of American Institute of Physics Conference Series, pp. 543-546, doi: 10.1063/1.58809, 1999.

Veltri, P., Nigro, G., Malara, F., Carbone, V., and Mangeney, A.: Intermittency in MHD turbulence and coronal nanoflares modelling, Nonlin. Processes Geophys., 12, 245-255, doi:10.5194/npg-12-245-2005, 2005.

Zhou, Y., Matthaeus, W., and Dmitruk, P.: Colloquium: Magnetohydrodynamic turbulence and time scales in astrophysical and space plasmas, Rev. Mod. Phys., 76, 1015-1035, 2004. 